\title{
Network boundary origins of fast solar wind seen in the low transition region?
}

\author{
M. D. Popescu ${ }^{1,2}$, J. G. Doyle ${ }^{1}$, and L. D. Xia ${ }^{1}$ \\ 1 Armagh Observatory, College Hill, Armagh BT61 9DG, N. Ireland \\ e-mail: [mdp; jgd; xld]@star.arm.ac.uk \\ 2 Astronomical Institute of the Romanian Academy, 75212 Bucharest 28, Romania
}

Received 18 September 2003 / Accepted 3 March 2004

\begin{abstract}
We present a study of a high spatial resolution raster acquired on-disk with the SUMER (Solar Ultraviolet Measurements of Emitted Radiation) grating spectrograph on SoHO (the Solar and Heliospheric Observatory) in a polar Coronal Hole $(\mathrm{CH})$ region. We analyse two EUV emission lines, representing the properties of solar plasma in the low transition region (TR), O III $703.87 \AA$ (maximum electron temperature, $T_{\mathrm{e}} \approx 8 \times 10^{4} \mathrm{~K}$ ), as well as in the corona, Mg IX $706.02 \AA$ $\left(T_{\mathrm{e}} \approx 10^{6} \mathrm{~K}\right)$. For Mg IX, we find that low $\mathrm{CH}$ intensities correspond to negative Doppler velocities (outflows) of $\approx 5 \mathrm{~km} \mathrm{~s}^{-1}$. Along the quiet Sun (QS)/CH boundaries, the coronal plasma begins to be more red-shifted. A coronal bright point (BP) located within the $\mathrm{CH}$ is blue-shifted in the coronal line. In the TR line, the outer region of the BP is red-shifted at $\approx 5 \mathrm{~km} \mathrm{~s}^{-1}$, but, towards its middle, the shift is around zero. The $\mathrm{O}$ III line, although it shows predominant downward motion of $\approx 5.5 \mathrm{~km} \mathrm{~s}$ in the $\mathrm{CH}$ and $\approx 6 \mathrm{~km} \mathrm{~s}^{-1}$ in the QS, it also has blue-shifts arranged in a small-scale network pattern with average negative values of $3.5 \mathrm{~km} \mathrm{~s}^{-1}$ in $\mathrm{CH}$ and $3 \mathrm{~km} \mathrm{~s}^{-1}$ in the QS. The blue-shifts are caused either by plasma outflows of a few km s${ }^{-1}$, or by transient events such as bi-directional jets which dislocate plasma to upward velocities even higher than $100 \mathrm{~km} \mathrm{~s}^{-1}$. The outflows originate predominantly from the intersection between the magnetic network and the inter-network cells (network boundaries). The bi-directional jets are found along the $\mathrm{CH} / \mathrm{QS}$ boundaries, and, moreover, in locations where the plasma seen in the Mg IX line is blue-shifted, but very close to small red-shifted regions. Another interesting change in behaviour is observed at the QS/CH boundaries, in the O III line, where plasma from the network changes its velocity sign, becoming redshifted. Our results constitute the lowest-in-altitude observed signature of plasma outflows from the chromospheric network boundaries inside a $\mathrm{CH}$. We have derived this conclusion from direct correlation between Doppler velocity and the intensity of the O III $702.87 \AA$ A line.
\end{abstract}

Key words. Sun: UV radiation - Sun: corona - Sun: transition region - Sun: solar wind

\section{Introduction}

Suggestions that the fast solar wind might originate from magnetically "open" regions in the corona rather than from regions of elevated coronal temperatures were first made four decades ago (see e.g. Billings \& Roberts 1964; Davis 1965). Those regions with an open magnetic field were identified by Waldmeier (1951) using ground observations of the visible green Fe XIV $5303 \AA$ line, with a combination of a coronograph-spectrograph. He later named them "coronal holes" (Waldmeier 1957), but the previous name "M-regions" ("M" from "mysterious") was still in use for some years.

From the late 1960s to the early 1970 s, a series of rocket flights aimed to investigate solar plasma properties in the wavelength range of 300 to $2950 \AA$. Even if important information concerning identification of lines was gained, the spectral

Send offprint requests to: M. D. Popescu,

e-mail: mdp@star.arm.ac.uk resolution larger than $0.4 \AA$ did not allow measurement of shapes and broadenings and thus, information about plasma dynamics in the solar atmosphere was not obtained.

However, these measurements permitted the first identification of a coronal hole $(\mathrm{CH})$ as the source of a high velocity solar wind stream (Krieger et al. 1973). The result came by comparing soft X-ray on-disk data taken during a sounding rocket flight on 24 November 1970 in the bands from 3 to $35 \AA$ and from 44 to $51 \AA$ with the wind velocity measured near the Earth by the Vela and Pioneer VI and VII spacecraft. Using the method of instantaneous ideal spirals, the wind bulk velocity was extrapolated back to the southern hemisphere $\mathrm{CH}$ region.

The next generation of UV spectrographs in space had higher spatial and spectral resolution, allowing the first measurement of plasma velocities in the solar atmosphere. These spectroscopic studies were based mainly on observations taken with the S082-B EUV spectrograph from Skylab ATM (Apollo Telescope Mount), as well as with a few high resolution 
EUV spectrometers which flew on rockets or space missions for short-time intervals, providing therefore only scarce data. The Skylab orbiting satellite, launched on 14 May 1973 was manned by three different crews until 8 February 1974.

The Solar and Heliospheric Observatory (SoHO), launched in 1995 , brought new insight to the physics of plasma outflows. Previous observations, though they brought important results concerning the dynamics of the transition region (TR) and corona, they did not have sufficient resolution for resolving small-scale structures.

We begin with a review of the previous work on determining the fast solar wind origins, before the advent of SoHO, as well as the results acquired with the SUMER instrument (Sect. 2). The results from our analysis are given in Sect. 3 with a summary in Sect. 4. The last section contain comments and conclusions hopefully increasing the knowledge about the origins of the fast solar wind, as the obtained results might indicate the lowest-in-altitude observed signature of plasma outflows from the chromospheric network boundaries inside a $\mathrm{CH}$.

\section{Searching for the fast solar wind origins}

Krieger et al. (1973) finally revealed the mystery of the $\mathrm{CHs}$, as they proved that previous suggestions of the fast solar wind originating from magnetically open regions were correct. For a more precise analysis of mass motions and other diagnostics (such as temperatures, densities, emission measures and elemental abundances) in the chromosphere, TR and corona, higher resolution spectral observations in the UV were needed.

\subsection{Early observations}

Measurements of TR Doppler shifts were first performed using data from the NRL normal-incidence spectrograph on Skylab, S082-B (Doschek et al. 1976). All wavelength shifts detected for ions with formation temperature higher than $7 \times 10^{4} \mathrm{~K}$ were red-shifted (measured relative to the chromosphere) with the largest velocities being around $15 \mathrm{~km} \mathrm{~s}^{-1}$. The net motions appeared to cease at $2.5 \times 10^{5} \mathrm{~K}$, as seen in the O V $629 \AA$ line. The instrument had $0.06 \AA$ spectral and $2^{\prime \prime}$ spatial resolution, sufficient to infer that the motions were primarily network phenomena. The interpretation of the results is that either the regions moving toward the chromosphere are brighter than the regions moving toward the instrument, or there may be more regions moving toward the chromosphere than away from it.

The results of Nolte et al. (1976) indicated that all large, near-equatorial $\mathrm{CHs}$ seen during Skylab flights are sources of high-speed solar wind as measured in the ecliptic plane at $1 \mathrm{AU}$, with the interplanetary magnetic polarity in the stream agreeing with the solar polarity beneath the hole. Other studies that confirmed $\mathrm{CH}$ origins of the fast solar wind were done from sounding rocket observations carried out since 1973. The data collected on 30 August 1973 (with spectral resolution of $0.02 \AA$ and spatial resolution of $20^{\prime \prime}$ ) indicated an outflow from the CHs of $16 \mathrm{~km} \mathrm{~s}^{-1}$ as inferred from the coronal Si XI $303 \AA$ A, Mg IX $368 \AA$ and Mg X $610 \AA$ lines (Cushman $\&$ Rense 1976).
Higher resolution observations followed ( $1^{\prime \prime}$ spatial, $0.03 \AA$ spectral), as for example another rocket-borne EUV spectrometer that flew on 5 June 1979 (Rottman et al. 1981) and 15 July 1980 (Rottman et al. 1982). A major result of Rottman et al. (1981), confirmed by their later study, was the detection of an apparent systematic outflow, relative to the quiet Sun (QS), of $\approx 3 \mathrm{~km} \mathrm{~s}^{-1}$ average and $5 \mathrm{~km} \mathrm{~s}^{-1}$ maximum in the O v $629 \AA$ line, underlying a well-defined low-latitude $\mathrm{CH}$. Hence, it was observed that the origins of the solar wind could be found low in the TR and not in the corona as previously thought.

Observations were continued with the NRL High Resolution Telescope and Spectrometer (HRTS; $0.1 \AA$ spectral, 1" spatial resolution), a UV instrument that flew 10 times on rockets from 21 July 1975, as well as on the Spacelab 2 mission, from 29 July to 6 August 1985 . The goal of the 6th HRTS rocket flight, launched on 20 November 1988, was to observe a CH on disk in the C IV 1548/1552 A doublet (Dere et al. 1989). This observation showed that there are significantly more regions of outflow in the $\mathrm{CH}$ than in the QS although a net down-flow was detected in both regions. The average velocity found in the blue-shifted regions inside the $\mathrm{CH}$ (for $\mathrm{C} \mathrm{IV}$ ) was $5 \mathrm{~km} \mathrm{~s}^{-1}$.

From the connection between coronal and interplanetary measurements at mid latitudes by in situ Ulysses observations around solar minimum, Woo \& Habbal (1997) concluded that the fast solar wind originates not only from $\mathrm{CHs}$ but also from the QS. However, a clear spectroscopic evidence of such an outflow has not yet been found.

\subsection{SUMER on-disk $\mathrm{CH}$ studies}

Recent observations of SoHO UV spectrometers have greatly improved our knowledge about the radiation emission at different levels in the solar atmosphere. In particular, the SUMER (Solar Ultraviolet Measurements of Emitted Radiation) grating spectrograph on SoHO is specially designed to study the dynamical aspects of solar plasma. The instrument has been described in detail by Wilhelm et al. (1995) before launch, and by Wilhelm et al. (1997) and Lemaire et al. (1997) after the first months of operation.

The good spatial, spectral and temporal resolution of this instrument allows the determination of the shift and broadening of a large number of lines (more than 1000, some of them recorded for the first time). The wavelength ranges of SUMER are: for detector A, 390-805 $\AA$ (2nd order) and 780-1610 $\AA$ (1st order); for detector B, 330-750 $\AA$ (2nd order) and 660-1500 $\AA$ (1st order), however in the range below $500 \AA$ the sensitivity is low. One thus has access to information about the dynamical phenomena displayed by the emitting plasma from a broad range of atmospheric heights.

SUMER allows for the first time the study of small-scale plasma structures (down to $1^{\prime \prime} \approx 715 \mathrm{~km}$ on the Sun) from the high chromosphere to the low corona $\left(1-1.6 R_{\text {Sun }}\right)$, as well as its dynamics (at a temporal scale as low as a few seconds). It investigates EUV and FUV Doppler flows down to $1 \mathrm{~km} \mathrm{~s}^{-1}$, in a temperature range of $10^{4}$ to $2 \times 10^{6} \mathrm{~K}$. 
Two different approaches for looking at the dynamical properties of $\mathrm{CHs}$ are being used: on-disk and off-limb observations. Here we concentrate on the first type of data, summarising the state-of-the-art research performed on the solar disk in $\mathrm{CH}$ regions, which aims at determining the origins of the high velocity plasma outflows from the solar atmosphere. As regards off-limb investigations, the main difficulty is in distinguishing the true site from which the fast wind emanates: plumes or inter-plumes. Even the most recent investigations give contradictory results (see e.g. Teriaca et al. 2003; Del Zanna et al. 2003; Gabriel et al. 2003).

Using on-disk observations from SUMER, Warren et al. (1997) measured Doppler wavelength shifts in a North Pole CH in the O VI 1032/1038 $\AA$ and C II 1036/1037 $\AA$ lines. Their measurements indicated the presence of an average net redshift in $\mathrm{CHs}$ at temperatures of less than $3 \times 10^{5} \mathrm{~K}$. Measurements of systematic wavelength shifts in the Ne VIII 770/780 ̊ resonance lines relative to the QS indicated a transition to average outflows near $6.3 \times 10^{5} \mathrm{~K}$ in the $\mathrm{CH}$.

Hassler et al. (1999) suggested that the $\mathrm{CH}$ plasma outflow originates predominantly along chromospheric network boundaries, with the strongest outflows occurring at the intersections of network boundaries. The study was based on SUMER observations in lines from the chromosphere ( $\mathrm{Si}$ II $1533 \AA$ ), TR (C IV $1548 \AA$ ) and corona (Ne VIII $770 \AA$ A). The Ne VIII line revealed the first two-dimensional coronal images showing velocity structure in a $\mathrm{CH}$, suggesting that the solar wind emanates from localised regions along boundaries and boundary intersections of magnetic network cells.

Peter (1999a) investigated statistical properties of the C IV $1548 \AA$ and Ne VIII $770 \AA$ profiles seen in SUMER fulldisk observations. His results indicated for both the Southern and the Northern CHs a net blue-shift in the Ne VIII line, while in the case of C IV, the holes seem to be blue-shifted compared to the QS, having a smaller outflow value than the Ne VIII line.

Teriaca et al. (1999) examined emission-line profiles observed on-disk with SUMER, from the low chromosphere to the corona and found that for both the quiet and active Sun the line shift from red to blue occurred at electron temperatures of $\approx 5.5 \times 10^{5} \mathrm{~K}$. Peter \& Judge (1999) found the same transition temperature at disk centre. The authors presented evidence for an outflow of the fast solar wind from CHs throughout the whole TR. These results imply that the fast wind in CHs would start with a low velocity in the upper chromosphere and lower TR (C II, C IV, N V), then decreasing in the middle TR (O IV, S VI, O v), before, finally, accelerating from the upper TR (O VI, Ne VIII). Peter \& Judge (1999) found CH blueshifts also in the He I $548 \AA$ line, but, as discussed in Peter (1999b), this may not be caused by a real mass flow. Instead, Peter (1999b) indicates a radiative transfer effect mainly causing the He I blue-shift.

The source and acceleration of the fast solar wind was also investigated by Dammasch et al. (1999), using SUMER data. For Ne VIII, they deduced outflows of $\approx 9 \mathrm{~km} \mathrm{~s}^{-1}$ in $\mathrm{CH}$ regions, and of $1 \pm 1 \mathrm{~km} \mathrm{~s}^{-1}$ in QS areas.

Stucki et al. (1999, 2000b) presented an analysis of a sample of SUMER FUV emission lines. When comparing line intensities, shifts and widths in $\mathrm{CHs}$ with the corresponding values obtained in the QS, they found that with increasing formation temperature, the spectral lines show on average an increasingly stronger blue-shift in $\mathrm{CHs}$ relative to the $\mathrm{QS}$ at equal heliospheric angle. The coolest lines in the sample (formation temperature $\approx 10^{4} \mathrm{~K}$ ) indicated a small relative red-shift. With respect to the rest wavelength, however, only lines formed above $5 \times 10^{5} \mathrm{~K}$ show blue-shifts in $\mathrm{CHs}$, which is not very different from the QS. The width of the lines is generally larger (by a few $\mathrm{km} \mathrm{s}^{-1}$ ) inside the CH. Stucki et al. (2000a) also observed that Ne VIII $770 \AA$ A showed an increasing blue-shift with increasing intensity of N IV $765 \AA$ in both QS regions and CHs.

Wilhelm et al. (2000) reported that the source of the fast solar wind in polar CHs can clearly be seen in the Ne VIII line of the low corona, either as dark polar caps in radiance diagrams or as regions of predominant blue-shift. However, for the chromospheric He I $584 \AA$ line they did not decide whether or not their data indicated the effects of mass flows. The average blue-shifts along the line of sight (LOS) in CHs amount to velocities of $\approx 3 \mathrm{~km} \mathrm{~s}^{-1}$ for both He I and Ne VIII, if the Doppler shift can be interpreted as an indication of bulk motions. The Ne VIII LOS velocity only in regions with strong outflow is $10 \mathrm{~km} \mathrm{~s}^{-1}$.

Xia et al. (2003) did an analysis concerning the source of the fast solar wind by comparing Doppler-shift maps of Ne VIII with charts of the photospheric magnetic field (NSO/Kitt Peak magnetograms) in an equatorial $\mathrm{CH}$. The larger blue-shifts of the Ne VIII line were associated mainly with the darker region, where the strong magnetic flux with a single polarity is concentrated. Conversely, the smaller blue-shifts were measured mainly in the brighter region, with an underlying mixedpolarity magnetic structure. These observational results are in agreement with the model prediction that the fast solar wind is initially accelerated in the coronal funnels, which are regions with open coronal fields rooted in the magnetic network.

\section{Our investigation of a SUMER raster}

It is well established that line profiles, through the Doppler effect, can provide us with vitally important clues to the dynamical structure of the solar atmosphere. We will concentrate mainly on determining the line shifts and widths in CHs situated in polar regions. From this data we will determine the plasma velocities, with the aim of observing how they correlate with the small-scale features inside $\mathrm{CHs}$ at different solar atmospheric heights.

The first data set to which we devoted our attention is a raster taken in a northern Pole $\mathrm{CH}$ region (on-disk) on 17 March 1999, with detector B from SUMER.

The "specific" spectrum of the considered raster (the intensity, in counts vs. the spectral range, in $\AA$, for all the spatial pixels summed along both solar axes) is plotted in Fig. 1. It covers a wide (512 pixels) spectral region: 697.5-720.5 (in 1st order). The emission lines from this spectral interval were identified from the SUMER spectral atlas of Curdt et al. (2001). As most of them are either blended or have a very low amplitude, we have chosen to study the characteristics of two lines: O III 703.87 $\AA$ and Mg IX 706.02 $\AA$. As seen in the CHIANTI database, the O III feature at $703.87 \AA$ contains 


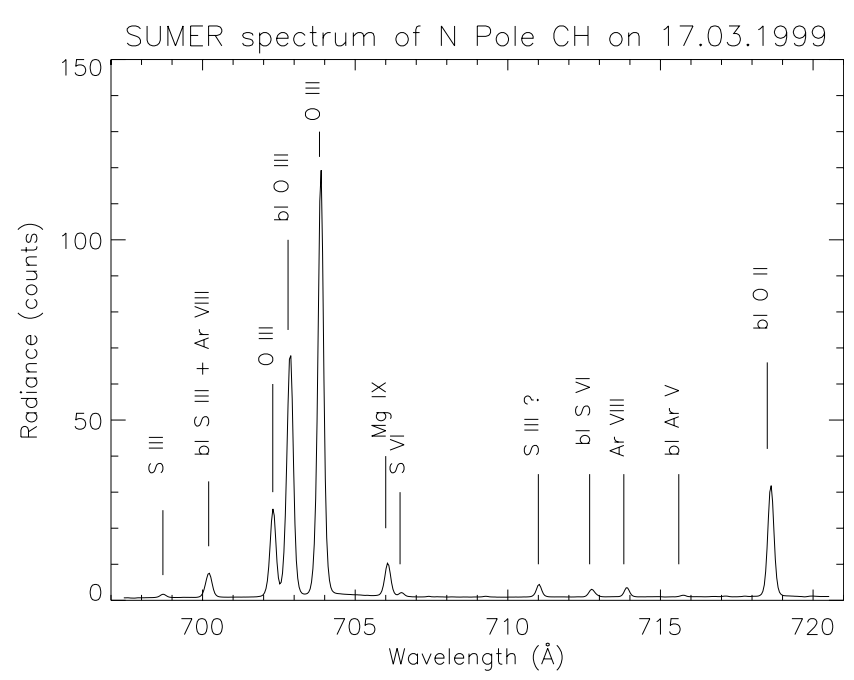

Fig. 1. The "specific" CH spectrum of 17 March 1999 taken with detector B of SUMER. The spectral range extends from 697.5 to $720.5 \AA$ (in 1st order). The O III $703.87 \AA$ line is the predominant feature of the on-disk data; the coronal Mg IX $706.02 \AA$ line appears much weaker, but we had determined its properties after a spatial binning.

two lines, but the separation between them is small $(5 \mathrm{~m} \AA$, corresponding to $\approx 0.1 \mathrm{~km} \mathrm{~s}^{-1}$, much below SUMER velocity uncertainty). The approximative formation temperature in ionisation equilibrium is $8 \times 10^{4} \mathrm{~K}$ for the $\mathrm{O}$ III line and $10^{6} \mathrm{~K}$ for Mg IX (Mazzotta et al. 1998).

The relatively weak intensity of the coronal line does not allow a high spatial resolution analysis. Because the TR line is strong, the signal to noise ratio is good, and no spatial binning was needed. For O III, therefore, we were able to keep the high resolution of $\approx 1^{\prime \prime}$. In this way, we have been able to identify the fine structures from the low TR, which, so far, constitute the key for our most important findings.

The data used for this study consist of 97 individual exposures taken with the $1^{\prime \prime} \times 300^{\prime \prime}$ slit. The characteristics of the raster are as follows: spectral resolution elements of $22.4 \mathrm{~m} \AA$, pixel size 1.046" and slit increment step 1.125". The data were taken during 00h 01m-04h 02m UT. Each spectrum has an integration time of $150 \mathrm{~s}$. One spectrum (the 14th) is missing, thus we eliminated it from our study. In physical units, the raster dimensions are $108^{\prime \prime}$ on the solar $x$ and $292^{\prime \prime}$ on the solar $y$ axis.

The raster location is given in Fig. 2, as seen in the EIT (Delaboudiniere et al. 1995) Fe XII $195 \AA$ image. As well as using the information given in the SUMER headers, the position of the raster was determined with visual identification techniques. In particular, we used as reference the detected coronal bright point as seen in our Mg IX line and the EIT Fe XII image.

\subsection{Data calibration}

As the total counting rate was not higher than 50000 counts $\mathrm{s}^{-1}$, we did not need to apply the correction for the dead-time effect, nor did we use the radiometric calibration, so the measurement remained in counts $\mathrm{px}^{-1} \mathrm{~s}^{-1}$. The flat field used to correct the images was taken on 18 March 1999.

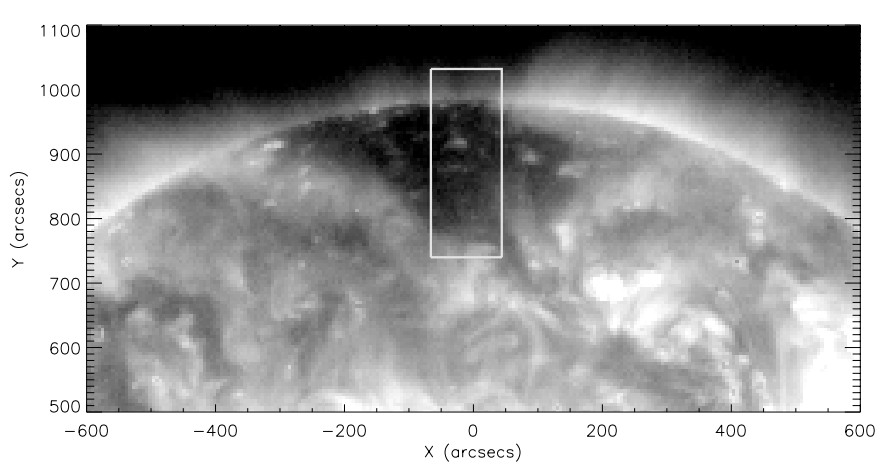

Fig. 2. The SUMER raster location in the North Pole $\mathrm{CH}$ of 17 March 1999, seen on the EIT Fe XII $195 \AA$ A (courtesy of the EIT consortium).

The geometrical distortion was corrected, thus straightening the slit images and aligning them with the image columns.

For a more detailed description about the necessity of the data calibration see Teriaca et al. (1999). Ultimately, we applied standard procedures for SUMER fts file handling.

\subsection{Binning, Gaussian fitting and additional corrections}

SUMER has no on-board wavelength calibration, thus appropriate attention needs to be paid in order to designate an absolute wavelength scale for the spectra. Usually, this is done using chromospheric lines of neutral atoms, but in our case we did not have any of those in the spectral interval. To calculate the dispersion, we took in the first instance the two O III lines (702.33 $\AA$ and $703.87 \AA$ ).

The pixel position was found by applying a Gaussian fit to the specific (binned) spectrum, that giving us a good signal-tonoise ratio for the entire $\mathrm{CH}$ raster. We converted the pixel to $\AA$ by taking O III $703.87 \AA$ as the reference line.

For the Mg IX line, the signal was not high enough with respect to the background noise, so we had to perform a $(4 \times$ 4) pixel binning on the spatial solar $x$ and solar $y$ coordinates.

Considering a Gaussian profile a good approximation for TR and coronal lines, we applied a fitting procedure through which we gained information about their amplitude, central position and the full width at half maximum ( $F W H M)$. We also corrected the measured $F W H M$ for the contribution of the instrumental profile, using the con-width-funct-3.pro routine based on the work of Chae et al. (1998).

We made an additional set of three corrections for the final velocity values. When summing the positions on the solar $y$ slit, we noticed a residual displacement of the line centre, which had to be corrected. This is a thermal effect as noted by Dammasch et al. (1999). When summing the positions on the solar $x$ direction, we also noticed the high spatial frequency variations, which are of instrumental origin, but are not solved by the geometrical correction (see Peter \& Judge 1999, for a more detalied discussion). For both those corrections we followed the same method as described by Dammasch et al. (1999). 
Finally, we obtained a pixel-to-wavelength relationship for the O III $703 \AA$ and Mg IX $706 \AA$ A lines, assuming a zero shift at the limb.

The uncertanity in determining the velocity includes the fitting error $\left(0.1^{\prime \prime} \approx 2 \mathrm{~km} \mathrm{~s}^{-1}\right)$, the geometrical error from the standard calibration $\left(2 \mathrm{~km} \mathrm{~s}^{-1}\right)$, as well as a subjective error in our estimation of the limb velocity that was substracted from the data. We threfore estimate an overall velocity uncertanity of about $3 \mathrm{~km} \mathrm{~s}^{-1}$.

\section{Results}

From the characteristics of the fitted line profiles we derived the intensities and the Doppler (LOS) velocities, as:

$v=\frac{\left(p_{i}-p\right) d}{\lambda_{\mathrm{th}}} \cdot c$,

and

$I=\frac{\sqrt{\pi}}{2 \sqrt{\ln 2}} \cdot I_{0} \cdot F W H M$,

where $p_{i}$ is the measured pixel position of the line centre in each coordinate, $p$ is the line centre of the specific spectrum, $d$ is the dispersion, $\lambda_{\text {th }}$ is the theoretical wavelength (from the SUMER spectral atlas of Curdt et al. 2001), $c$ is the light velocity and $I_{0}$ is the measured line maximum.

After correcting for the instrumental broadening, and assuming that the line studied is optically thin, the measured full width at half maximum is:

$F W H M=2 \sqrt{\ln 2} \frac{\lambda_{i}}{c} \sqrt{\frac{2 k_{\mathrm{B}} T_{i}}{M_{i}}+\xi^{2}}$,

where $\lambda_{i}$ is the measured wavelength, $k_{\mathrm{B}}$ the Boltzmann constant, $T_{i}$ the ion temperature, $M_{i}$ the ion mass, and $\xi$ is the nonthermal broadening.

The intensity and LOS velocity maps are given in the middle plots of Fig. 3. The spatial resolution is $1^{\prime \prime}$ for the TR line and 4 " for the coronal line. The dimension of the images (solar $x$ and $y$ ) is given in arcseconds. The zero point does not represent the real zero solar coordinate, but is given relative to the starting point of our raster. The images in Fig. 3 are rotated by $90^{\circ}$, so that the limb (situated at solar $y \approx 285$ for the $\mathrm{Mg}$ IX line, $\approx 245$ for the $\mathrm{O}$ III line and $\approx 240$ in the continuum) is now toward the right part of the plots.

If we had to perform a spatial binning on the $\mathrm{O}$ III line, the features observed both in the intensity and Doppler velocity would dramatically loose their clear appearance as seen in the $\approx 1^{\prime \prime}$ resolution. The binning that we performed for the $\mathrm{Mg}$ IX line was not so harmful, as the coronal features do not have such a fine spatial structure as in the low TR.

The outflows of plasma from the magnetic network boundaries in the $\mathrm{CH}$, together with dynamical characteristics of other atmospheric phenomena, are revealed in Fig. 3. We discuss these features in more detail in the following subsections.

\subsection{The two-dimensional plots}

The intensity of the emitted light in our raster reveals plasma structures characteristic of the corona, as seen in the Mg IX line, and of the low TR, in the O III line (Fig. 3, middle plots). For the LOS velocities, negative values (blue) denote blue-shifts (upward plasma motion), while positive values (plotted in red) are red-shifts (downward motion); zero shifts are shown in white.

In the coronal line, the $\mathrm{CH}$ is clearly seen as a reduction in intensity, surrounded by the brighter QS. The contours on the plot indicate the QS/CH boundaries. We also notice a coronal bright point (BP) with an approximate dimension of $20^{\prime \prime} \times 20^{\prime \prime}$. The outflow motion has an average velocity of $\approx-5 \mathrm{~km} \mathrm{~s}^{-1}$, well correlated with the $\mathrm{CH}$ low intensities. Several regions inside the $\mathrm{CH}$ have outflows with typical values of $\approx-10 \mathrm{~km} \mathrm{~s}^{-1}$. The red-shifts (average value $\approx 1 \mathrm{~km} \mathrm{~s}^{-1}$ ) are seen more in the small QS portion than inside the $\mathrm{CH}$. The mean Doppler velocity inside the $\mathrm{CH}$ is $\approx-4.5 \mathrm{~km} \mathrm{~s}^{-1}$, while along the QS region it is $\approx-3.5 \mathrm{~km} \mathrm{~s}^{-1}$.

The bright magnetic network is clearly seen in the TR line intensity, and there is no distinguishable difference in its appearance between the $\mathrm{CH}$ and the QS. In the velocity map the downward motion is predominant, but the blue-shifted plasma forms a small-scale network pattern with average negative values of $3.5 \mathrm{~km} \mathrm{~s}^{-1}$ in $\mathrm{CH}$ and $3 \mathrm{~km} \mathrm{~s}^{-1}$ in the QS.

The average value of the $\mathrm{O}$ III line Doppler shift is $\approx 3.5 \mathrm{~km} \mathrm{~s}^{-1}$ in the $\mathrm{CH}$ and about $4.5 \mathrm{~km} \mathrm{~s}^{-1}$ in the QS regions. The downward motion only is $5.5 \mathrm{~km} \mathrm{~s}^{-1}$ in the $\mathrm{CH}$ and slightly higher in the QS.

In Fig. 4 we plot the outflow contours (values $\leq-3 \mathrm{~km} \mathrm{~s}^{-1}$ ) against intensity, for a portion of the raster in the $\mathrm{O}$ III line. The upward motions are found mostly at the chromospheric network boundaries, and they are more in the $\mathrm{CH}$ than in the QS. Through network boundary we understand the intersection between the magnetic network and the inter-network cells.

Because the features are small, it is difficult to see how plasma moves in relation to its intensity. One-dimensional cuts at different positions on the solar $x$ axis show more clearly the correlation between the Doppler velocity and the intensity for the O III line (see Fig. 3).

\subsection{The one-dimensional plots}

We give as examples two solar $x$ positions of the raster in the Mg IX line and six in O III, plotting the intensity and the Doppler velocity along the solar $y$ direction. There are more examples given for the TR line because the features seen in this region are much more variable than in the corona on a small spatial scale. The selected cuts are: position 8 on Mg IX solar $x$, followed by positions 8,9 and 10 on the O III line (left plots) and solar $x=57$ on Mg IX, followed by positions 57, 58 and 59 on the O III solar $x$ axis (right plots). The location of the cuts is also given in the Mg IX intensity map.

The intensity is plotted as a dashed line and the Doppler velocity in blue (values $\leq-1.5 \mathrm{~km} \mathrm{~s}^{-1}$, upward motion), red (values $\geq 1.5 \mathrm{~km} \mathrm{~s}^{-1}$, downward motion), and white (zero shift), with the corresponding relative uncertainty of $\approx 3 \mathrm{~km} \mathrm{~s}^{-1}$. 

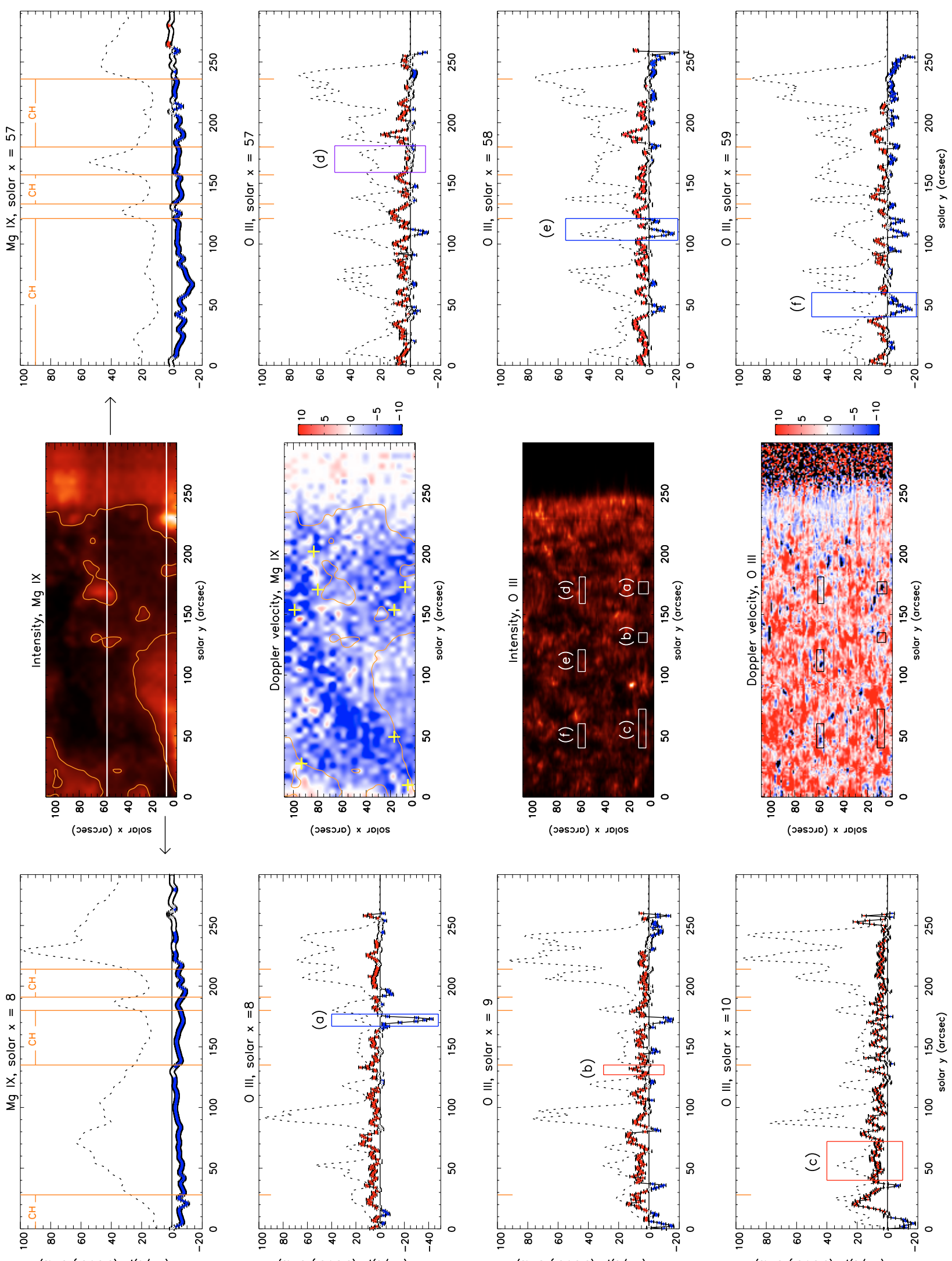

Fig. 3. Sides: Mg IX and O III intensity (dotted line) and Doppler velocity (the blue/red symbols with error bars) for the selected solar $x$ cuts. Orange lines indicate the QS/CH boundary. The features identified are: a bi-directional jet a); an inter-network cell b); QS chromospheric network c); a coronal BP d); $\mathrm{CH}$ network e) and f); Center: intensity and Doppler velocity maps for $\mathrm{Mg}$ IX (with the orange $\mathrm{CH}$ contour and the white lines indicating, with arrows, the displayed cuts) and O III (with the selected features). The " + " signs in the Mg IX velocity map indicate the locations of identified bi-directional jets, as seen in the $\mathrm{O}$ III line. 


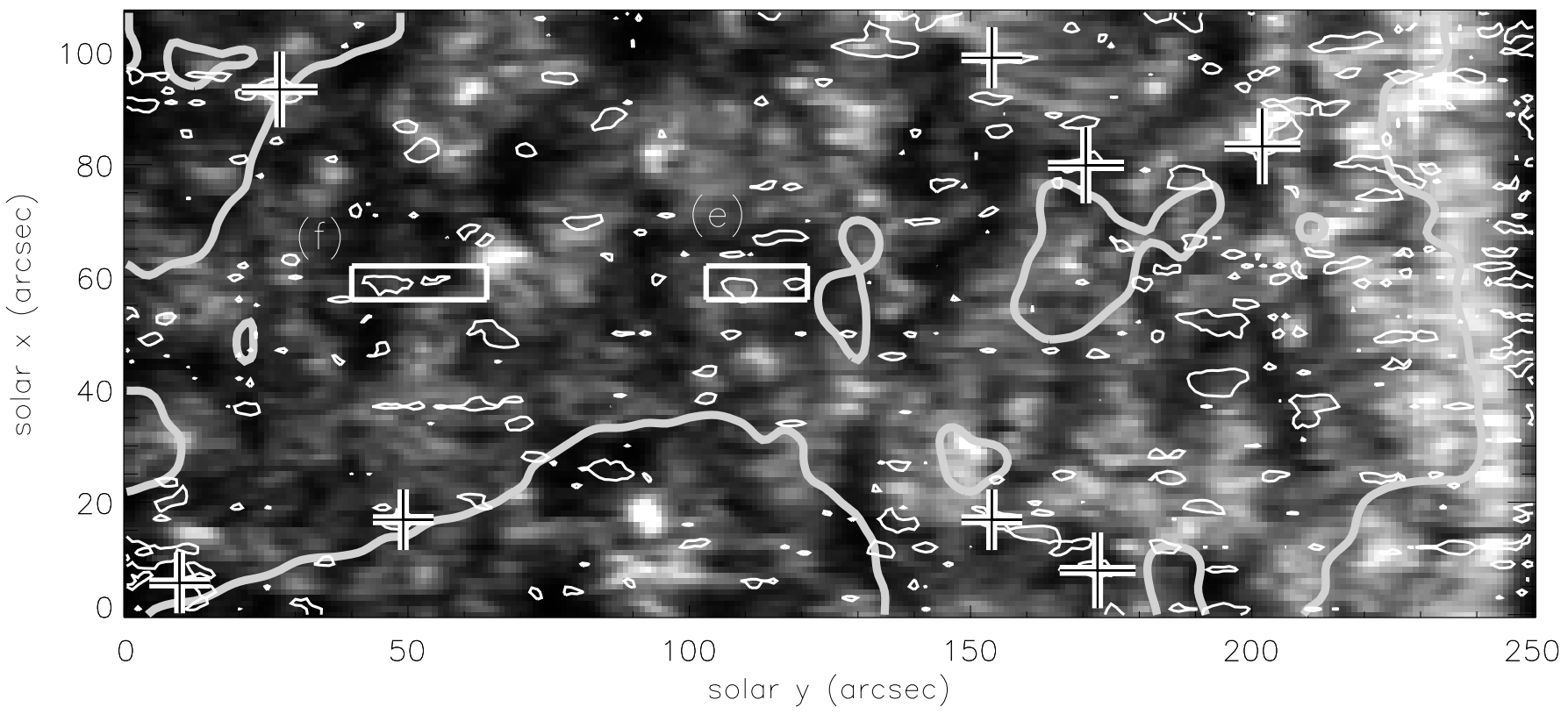

Fig. 4. O III $703 \AA$ outflows (velocities $\leq-3 \mathrm{~km} \mathrm{~s}^{-1}$; thin white contours) as seen against the O III intensity, from $0^{\prime \prime}$ up to $250^{\prime \prime}$. The thick grey coutour represents the QS/CH boundaries from the the Mg IX $706 \AA$ line. We also indicate the features e) and f) which we selected as examples of outflows. The "+" signs indicate the bi-directional jets, with a clear double Gaussian structure over a few spatial pixels. The displayed magnetic network boundary blue-shifts indicate the outflows of plasma which might constitute the origins of the fast solar wind.

\subsection{Feature classification}

For a closer examination, we selected five types of phenomena labeled (a)-(f) in Fig. 3, as discussed below.

\subsubsection{Bi-directional jets}

In the bi-directional jet - feature (a) - plasma undergoes rapid movements. Another term defining these phenomena is "explosive events". Their spectra usually show both blue and red shifted wings, but in these data one can see mainly the blueshifted component, possibly because the integration time is long (150 s), and their blue-shifted component is, most of the times, predominant (Madjarska \& Doyle 2003). As seen in Fig. 5, the bi-directional jet spectrum shows a double peak structure, with a small increase in the intensity.

In Fig. 3 the velocity is given from the single Gaussian fit, which in this type of event is not appropriate, as it obviously gives very high errors. When fitting spectra from bi-directional jets with a double Gaussian, we found outflow LOS velocities of more than $100 \mathrm{~km} \mathrm{~s}^{-1}$. The red-shifted component of those jets is smaller, on the order of $10-20 \mathrm{~km} \mathrm{~s}^{-1}$, and the difference between the red- and the blue-shifted components can be as high as $150 \mathrm{~km} \mathrm{~s}^{-1}$.

Although our raster has an integration time too long for studying these short lived events, we were able to identify a few. We indicated them with "+" signs on the plots (see Figs. 3 and 4). There might be more, but we selected only the ones for which the double spectrum structure is clearly recognizable, for a few consecutive spatial pixels along the solar $y$ axis. The size of the selected examples of bidirectional jets is about 5 pixels on the solar $y$ direction (that means $\approx 3500 \mathrm{~km}$ ) and about $2-3$ pixels on the solar $x$ direction

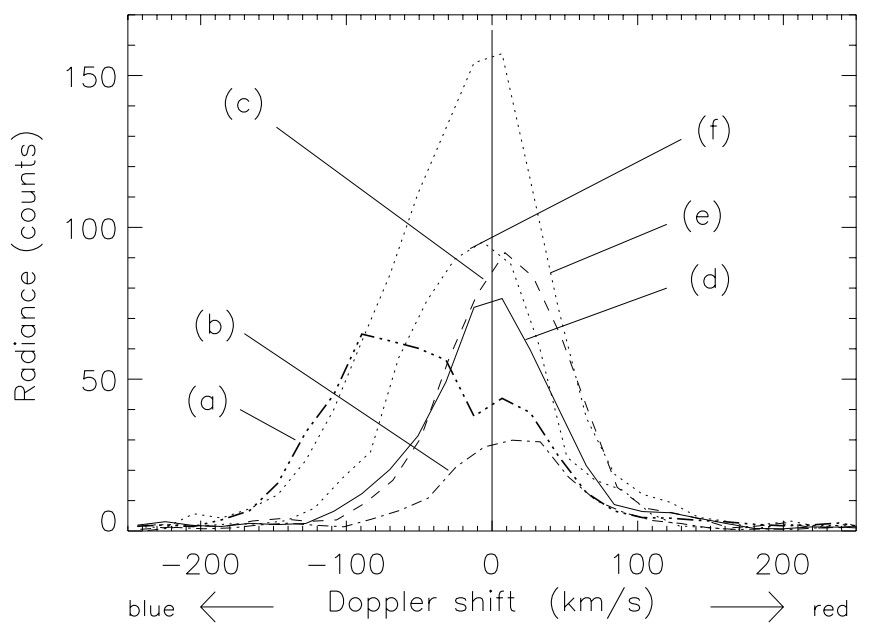

Fig. 5. Examples of spectra from the O III line, representing the features: a) a bi-directional jet; b) an inter-network cell red-shift; c) a QS network red-shift; d) a coronal BP; e) and f) $\mathrm{CH}$ network boundary blue-shifts.

(therefore, a lifetime of $\approx 300-350 \mathrm{~s}$ ). As we selected only the locations in which the double Gausssian structure of the spectrum is clear, we obviously selected only the biggest events, both in size and duration.

The identified bi-directional jets are found inside the $\mathrm{CH}$ along the $\mathrm{CH} / \mathrm{QS}$ boundary, and, moreover, in locations where the plasma seen in the Mg IX line is blue-shifted, but close to small red-shifted regions. This was recently observed for first time by Madjarska et al. (2004), in the N IV $765.15 \AA$ line. The jets are the signature of magnetic reconnection between the open magnetic $\mathrm{CH}$ lines and the closed QS loops, 
and are thought to represent evidence for the slow solar wind origins (Madjarska et al. 2004).

\subsubsection{Inter-network cells}

In the inter-network cells, plasma is generally red-shifted. For the selected example - feature (b) - the highest velocity is $\approx 11 \mathrm{~km} \mathrm{~s}^{-1}$, while the mean value over its whole spatial length is $\approx 5 \mathrm{~km} \mathrm{~s}^{-1}$.

\subsubsection{Chromospheric network in the QS}

For the QS, the magnetic network - feature (c) - seen as increases in the $\mathrm{O}$ III intensity, is generally red-shifted. In the selected example, whose spectrum is given in Fig. 5, the highest LOS velocity is $\approx 11 \mathrm{~km} \mathrm{~s}^{-1}$, with an average over the length of the feature indicating values of $\approx 7 \mathrm{~km} \mathrm{~s}^{-1}$.

In a few cases, the QS magnetic network is blueshifted. But what we define as QS is actually a small portion near the $\mathrm{CH}$, which on a larger scale is defined as a $\mathrm{CH} / \mathrm{QS}$ boundary.

\subsubsection{Coronal BP}

The coronal BP - feature (d) - is seen as an increase in the Mg IX intensity, which corresponds to a smaller relative increase in the $\mathrm{O}$ III intensity. From Fig. 3 one can also see that the BP leads to an increase in the Mg IX off-limb intensity, which may form, further on, a plume. In the O III line, two of the detected bi-directional jets are located just near the $\mathrm{BP}$ boundaries. In this line, the outer region of the BP is redshifted with around $5 \mathrm{~km} \mathrm{~s}^{-1}$, and, towards its middle, the shift is around zero. A similar behaviour is observed for the $\mathrm{Mg}$ IX line, but with a $4 \mathrm{~km} \mathrm{~s}^{-1}$ blue-shift in the middle of the BP.

Similar results for BP were found by Wilhelm et al. (2000), who reported zero or outward directed LOS velocities in the Ne VIII line, and by Madjarska et al. (2003), who observed that the BP is dominated by red-shifts (up to $\approx 10 \mathrm{~km} \mathrm{~s}^{-1}$ ) but in a small area it is blue-shifted. Madjarska et al. (2003) data was a high cadence temporal series in S VI $933 \AA$ (originating at an intermediate height between our two lines, corresponding to $\approx 2 \times 10^{5} \mathrm{~K}$ ). The authors also made a comparison with MDI magnetograms, showing that the blue-shifted region was located between the two magnetic polarities.

\subsubsection{Chromospheric network in the $\mathrm{CH}$}

Inside the $\mathrm{CH}$, in the $\mathrm{O}$ III line, every time there is an increase in the intensity, it corresponds to decreases in the velocity, which, generally, becomes negative. Sometimes this correlation is shifted a few pixels, revealing that the outflow takes place generally not on the magnetic network itself, but at its boundaries with the inter-network cells. We selected as typical examples of outflow the features labeled (e) and (f), whose blue-shifts have similar negative maximum values of $15 \mathrm{~km} \mathrm{~s}^{-1}$ and $6-7 \mathrm{~km} \mathrm{~s}^{-1}$ on average.
It is interesting to follow the feature (e) over a few consecutive spatial pixels, as shown in Fig. 3 in the plots from solar $x=57$ to 59 . It changes from a single structure, with a maximum in the intensity and a minimum in the blue-shift, to a three-component structure, with a blue-shift minimum for every intensity increase.

In case (f), the intensity is smaller, and the blue-shift occurs not only on the intensity increase, but also at the intersection between the network and the cell (the network boundary). The appearance of the boundary blue-shift profile is that of a single Gaussian (see the spectra given in Fig. 5). The CH network boundary blue-shifts generally occur on a smaller spatial scale $(\approx 5000 \mathrm{~km}$ on the Sun) than those observed for the bidirectional jets.

There are more examples of magnetic network boundary outflows than the two selected on the displayed onedimensional plots. Most of them occur inside the $\mathrm{CH}$. But one should be careful in defining them. For example, on the same plot where we selected feature (c), at solar $y=0$ to 15 , the large scale blue-shift, and the higher value, represents another bi-directional jet, with a double Gaussian spectrum.

\subsection{O III line spectra}

The fine structure of the $\mathrm{O}$ III features, and their shift toward the blue and red can also be viewed in Fig. 6. Their spectra noticeably vary even in succesive locations on the solar $x$ axis on the two sets of examples given, solar $x=8$ to 10 and 57 to 59. For an easier comparison with the plots from Fig. 3, on the sides we give the relative position of the solar $y$ axis.

\subsection{Non-thermal broadening}

Assuming the plasma is in thermal equilibrium, so that the electron and ion temperatures are the same, we calculated the nonthermal velocities for both O III $703 \AA$ and Mg IX $706 \AA$. An additional non-thermal component would suggest the presence of motions, in the form of propagating waves and/or turbulence.

After correcting the instrumental broadenings ( $F W H M$ ) by applying the con-width-funct-3.pro routine, we calculated the non-thermal velocities for different regions in the raster. For Mg IX, the average non-thermal velocities are $\approx 56 \mathrm{~km} \mathrm{~s}^{-1}$ above the limb, $\approx 45 \mathrm{~km} \mathrm{~s}^{-1}$ in the dark $\mathrm{CH}$ region and $\approx 41 \mathrm{~km} \mathrm{~s}^{-1}$ in the QS and the BP. For O III, the values are $\approx 51 \mathrm{~km} \mathrm{~s}^{-1}$ in the $\mathrm{CH}$ region and $\approx 48 \mathrm{~km} \mathrm{~s}^{-1}$ in the QS and BP. The fact that the line widths are generally larger (by a few $\mathrm{km} \mathrm{s}^{-1}$ ) inside the $\mathrm{CH}$ than in QS was also reported by various authors (Lemaire et al. 1999; Stucki et al. 2000b; Xia 2003).

The derived non-thermal velocities are much higher than those obtained for lines with similar formation temperature, recorded with detector A (Teriaca et al. 1999; Xia 2003). The data used here were recorded with the SUMER detector B, for which the theoretical instrumental broadening in the measured line profile was evaluated to about 2 pixels for the 1 " wide slit. However, a previous study by Chae et al. (1998) showed that the real contribution of the instrument is larger than that. 

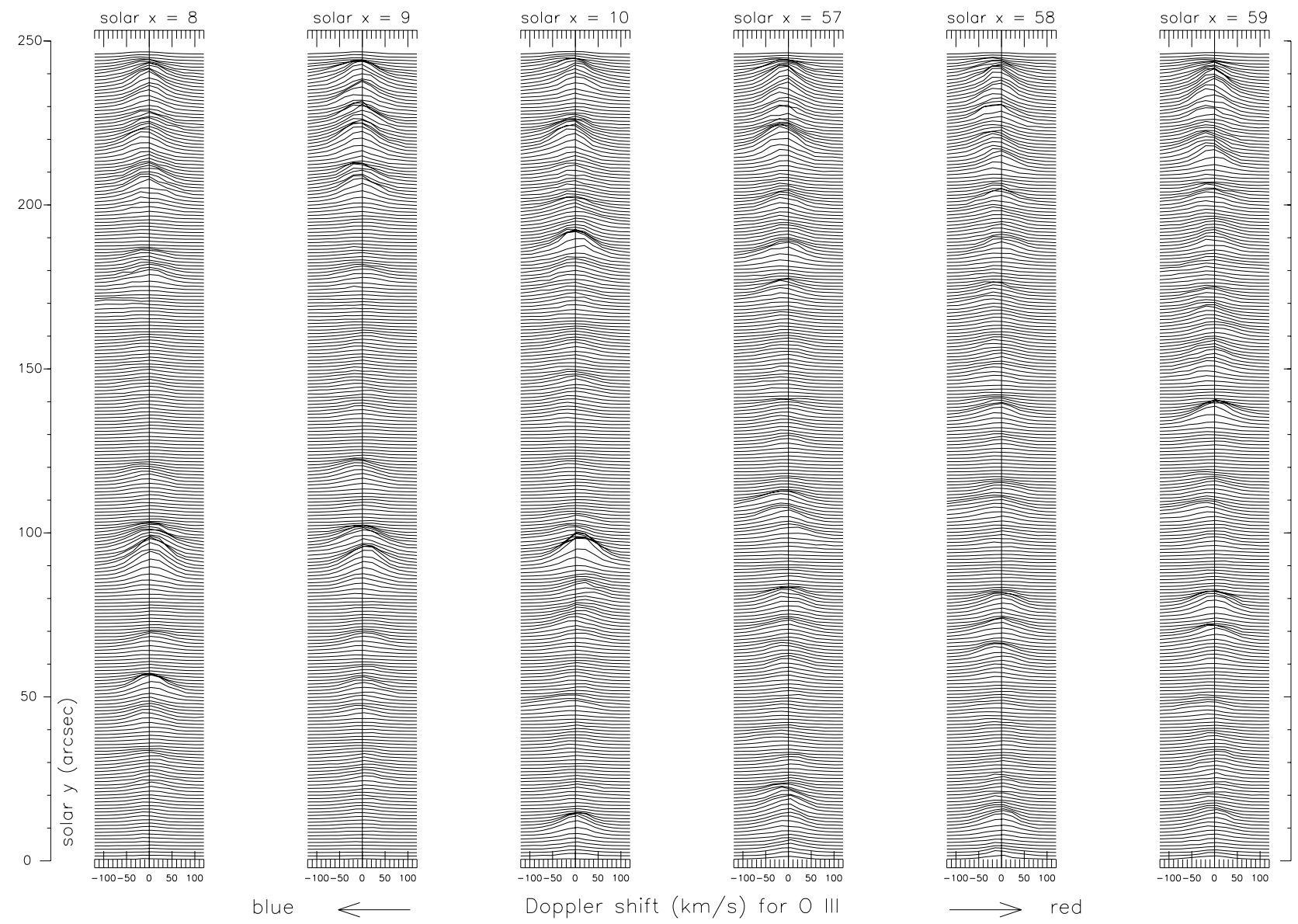

Fig. 6. Spectra of the O III line along the solar $y$ axis on the selected solar $x$ cuts. The line shift toward blue and red is easy to distinguish.

A value of $3 \pm 0.5$ pixels was suggested, which was adopted in the correction routine con-width-funct-3.pro. An even larger value of 4.1 pixels was proposed in a recent work of Moran (2003).

When we tried to correct the instrumental broadenings using the value suggested by Moran (2003), we found that the average non-thermal velocites for the $\mathrm{O}$ III line become smaller, i.e., $\approx 39 \mathrm{~km} \mathrm{~s}^{-1}$ in the $\mathrm{CH}$ and $\approx 35 \mathrm{~km} \mathrm{~s}^{-1}$ in the $\mathrm{QS}$ and $\mathrm{BP}$ regions. The $\mathrm{Mg}$ IX line has values of $\approx 45 \mathrm{~km} \mathrm{~s}^{-1}$ above the limb, $\approx 31 \mathrm{~km} \mathrm{~s}^{-1}$ in the $\mathrm{CH}$ region and $\approx 25 \mathrm{~km} \mathrm{~s}^{-1}$ in the QS and BP. We have obtained similar results when inspecting another data set taken the next day (18 March 1999) in the same CH. These values are comparable to the values found for other TR ions with detector A for equatorial $\mathrm{CH}$ (Xia 2003) and for QS (Teriaca et al. 1999; Xia 2003). The results support Moran's suggestion that a larger instrumental width should be adopted for detector $\mathrm{B}$.

\section{Discussion and conclusions}

We have analysed how plasma LOS motions correlate with the intensity features, in a $\mathrm{CH}$ region, at two different heights in the solar atmosphere, from a spectrum acquired with the SUMER spectrograph. In particular, we were mainly interested to see from where do the $\mathrm{CH}$ outflows originate.

For the coronal Mg IX $706 \AA$ line, the filling factor of blue-shifted material in the $\mathrm{CH}$ is about $90 \%$, with average
LOS velocity values of $\approx-5 \mathrm{~km} \mathrm{~s}^{-1}$. In some regions inside the $\mathrm{CH}$, the velocities are even higher, around $-10 \mathrm{~km} \mathrm{~s}^{-1}$ on average. The net LOS velocity in the QS is $\approx-4 \mathrm{~km} \mathrm{~s}^{-1}$.

In the case of the low TR O III $703 \AA$ line, the downward motion is predominant, with an average velocity of $5.5 \mathrm{~km} \mathrm{~s}^{-1}$ in the $\mathrm{CH}$, and $6 \mathrm{~km} \mathrm{~s}^{-1}$ in the QS. The red-shifted appearance of this line is sprinkled with blue-shifts forming a small-scale network pattern with average negative values of $3.5 \mathrm{~km} \mathrm{~s}^{-1}$ in $\mathrm{CH}$ and $3 \mathrm{~km} \mathrm{~s}^{-1}$ in the QS. The average value of the $\mathrm{O}$ III line Doppler shift is $\approx 3.5 \mathrm{~km} \mathrm{~s}^{-1}$ in the $\mathrm{CH}$ and $\approx 4.5 \mathrm{~km} \mathrm{~s}^{-1}$ in the QS.

In some case the blue-shifts are caused by high velocity transient events, such as bi-directional jets, which can dislocate plasma up to $100-150 \mathrm{~km} \mathrm{~s}^{-1}$. However, most of the upflows occur on a much reduced scale and a question remains as to whether these constitute a "steady flow" or are produced as a result of small scale transient features. The O III outflows are found mostly at the network intersection with the inter-network cells (network boundaries). Sometimes they occur on the magnetic network, but they generally have the tendency to avoid the brightest structures. The blue-shifts are more in the $\mathrm{CH}$ than in the QS. However, the way we defined the $\mathrm{CH} / \mathrm{QS}$ boundary is subjective, and our portion of QS is small, that on a larger scale could be defined as a CH/QS boundary. In the selected examples of $\mathrm{CH}$ outflows, the blue-shifts are around $-6 \mathrm{~km} \mathrm{~s}^{-1}$ (when averaged over a spatial scale of $7-8^{\prime \prime}$ ), but individual 
pixels can show values as high as $-15 \mathrm{~km} \mathrm{~s}^{-1}$. A time-series is required to throw further light on the variability of these flows.

In the inter-network cells, as seen in the TR line, plasma is usually red-shifted with $\approx 5 \mathrm{~km} \mathrm{~s}^{-1}$. The network outside the $\mathrm{CH}$ is also generally red-shifted with $\approx 7 \mathrm{~km} \mathrm{~s}^{-1}$. The detected BP corresponds, in this line, to a small portion of zero velocities, in the middle of the structure, surrounded by positive (upward) values of about $5 \mathrm{~km} \mathrm{~s}^{-1}$.

Our results are in accordance with the $\mathrm{CH}$ blue-shifts observed in coronal lines, and overall red-shifts for lower TR lines, with some regions of outflow, occuring more in $\mathrm{CH}$ regions than in the QS. This was revealed even before the SoHO era (see e.g. the results of Dere et al. (1989), derived from observations from a rocket flight). Previous works did provide strong evidence for the outflow of the fast solar wind from $\mathrm{CHs}$, but none of them found any correlation between the chromospheric network intensity and the Doppler velocity lower than for the coronal Ne VIII line. We think that this was due to the coarse spatial scale in which the shifts were determined.

The new result revealed by the present study is that we have seen regions of blue-shift in the $\mathrm{CH}$ network boundaries, which may represent the lowest-in-altitude observed origin of fast solar wind streams, if they are steady. As seen from the description of plasma behaviour in the $\mathrm{O}$ III line, it is the first time that motion in a $\mathrm{CH}$ at such a low atmospheric height (close to the base of the TR, $\approx 8 \times 10^{4} \mathrm{~K}$ ) is provided on a spatial scale small enough to distinguish its fine-scale correspondence with the intensity features.

Acknowledgements. We would like to acknowledge the referee, Werner Curdt, whose comments greatly helped in improving the study. We thank Ignacio Ugarte-Urra, Maria Madjarska and Ioannis Giannikakis for guidance with some IDL procedures, as well as Barrie Whittaker and Georgeta Mariş for their suggestions. Research at Armagh Observatory is grant-aided by the N. Ireland Dept. of Culture, Arts and Leisure. This work was supported in part by PPARC grant PPA/G/S/1999/00055 and by the Programme for Research in Irish Third Level Institutions for Grid-enabled Computational Physics of Natural Phenomena (CosmoGrid). The SUMER project is financially supported by DLR, CNES, NASA, and PRODEX. We also aknowledge Klaus Wilhelm who is responsible for taking the data we used.

\section{References}

Billings, D. E., \& Roberts, W. O. 1964, Astrophysica Norvegica, 9 , 147

Chae, J., Schühle, U., \& Lemaire, P. 1998, ApJ, 505, 957

Curdt, W., Brekke, P., Feldman, U., et al. 2001, A\&A, 375, 591
Cushman, G. W., \& Rense, W. A. 1976, ApJ, 207, L61

Dammasch, I. E., Wilhelm, K., Curdt, W., \& Hassler, D. M. 1999, A\&A, 346, 285

Davis, L. 1965, in Stellar and Solar Magnetic Fields, IAU Symp., 22, 202

Del Zanna, G., Bromage, B. J. I., \& Mason, H. E. 2003, A\&A, 398, 743

Delaboudiniere, J.-P., Artzner, G. E., Brunaud, J., et al. 1995, Sol. Phys., 162, 291

Dere, K. P., Bartoe, J.-D. F., Brueckner, G. E., \& Recely, F. 1989, ApJ, 345, L95

Doschek, G. A., Bohlin, J. D., \& Feldman, U. 1976, ApJ, 205, L177

Gabriel, A. H., Bely-Dubau, F., \& Lemaire, P. 2003, ApJ, 589, 623

Hassler, D. M., Dammasch, I. E., Lemaire, P., et al. 1999, Science, 283, 810

Krieger, A. S., Timothy, A. F., \& Roelof, E. C. 1973, Sol. Phys., 29, 505

Lemaire, P., Bocchialini, K., Aletti, V., Hassler, D., \& Wilhelm, K. 1999, Space Sci. Rev., 87, 249

Lemaire, P., Wilhelm, K., Curdt, W., et al. 1997, Sol. Phys., 170, 105

Madjarska, M. S., \& Doyle, J. G. 2003, A\&A, 403, 731

Madjarska, M. S., Doyle, J. G., Teriaca, L., \& Banerjee, D. 2003, A\&A, 398, 775

Madjarska, M. S., Doyle, J. G., \& van Driel-Gesztelyi, L. 2004, ApJ, 603, L57

Mazzotta, P., Mazzitelli, G., Colafrancesco, S., \& Vittorio, N. 1998, A\&AS, 133, 403

Moran, T. G. 2003, ApJ, 598, 657

Nolte, J. T., Krieger, A. S., Timothy, A. F., et al. 1976, Sol. Phys., 46, 303

Peter, H. 1999a, ApJ, 516, 490

Peter, H. 1999b, ApJ, 522, L77

Peter, H., \& Judge, P. G. 1999, ApJ, 522, 1148

Rottman, G. J., Klimchuk, J. A., \& Orrall, F. Q. 1981, ApJ, 247, L135

Rottman, G. J., Orrall, F. Q., \& Klimchuk, J. A. 1982, ApJ, 260, 326

Stucki, K., Solanki, S. K., Rüedi, I., et al. 1999, Ap\&SS, 264, 53

Stucki, K., Solanki, S. K., Schühle, U., \& Rüedi, I. 2000a, A\&A, 362, L49

Stucki, K., Solanki, S. K., Schühle, U., et al. 2000b, A\&A, 363, 1145

Teriaca, L., Banerjee, D., \& Doyle, J. G. 1999, A\&A, 349, 636

Teriaca, L., Poletto, G., Romoli, M., \& Biesecker, D. A. 2003, ApJ, 588, 566

Waldmeier, M. 1951, Die Sonnenkorona. I (Basel: Birkhäuser)

Waldmeier, M. 1957, Die Sonnenkorona. II (Basel: Birkhäuser)

Warren, H. P., Mariska, J. T., \& Wilhelm, K. 1997, ApJ, 490, L187

Wilhelm, K., Curdt, W., Marsch, E., et al. 1995, Sol. Phys., 162, 189

Wilhelm, K., Dammasch, I. E., Marsch, E., \& Hassler, D. M. 2000, A\&A, 353, 749

Wilhelm, K., Lemaire, P., Curdt, W., et al. 1997, Sol. Phys., 170, 75

Woo, R., \& Habbal, S. R. 1997, Geophys. Res. Lett., 24, 1159

Xia, L. D. 2003, Ph.D. Thesis (Göttingen: Georg-August-Univ.)

Xia, L. D., Marsch, E., \& Curdt, W. 2003, A\&A, 399, L5 